\title{
Mathematical Model of Cone Mosaic with Eye Optical Properties
}

\author{
Mio Kumagai, Yoshimi Kamiyama \\ Information Science and Technology, Aichi Prefectural University \\ 1522-3 Ibaragabasama, Nagakute, Aichi, Japan \\ kumagai@ nil.ist.aichi-pu.ac.jp; kamiyama@ist.aichi-pu.ac.jp
}

\begin{abstract}
Cone photoreceptors convert light into electrical signals and show maximum light sensitivity to either long (L), medium $(\mathrm{M})$, or short (S) wavelengths. Anatomically, the cones form a distinctive spatial arrangement on the retina, i.e., a cone mosaic. The Scones have specific sampling distributions; S-cones are rare $(<10 \%)$ and show non-random distribution. Their optical properties are thought to change the quality of retinal images. However, the influence of physiological aberrations on vision has not been fully explained. We developed a mathematical model describing a cone mosaic and the associated eye optics, based on the anatomical and physiological characteristics of cones. In the simulation, we demonstrated that the S-cones obtain a retinal image, despite their sparse distribution. The simulation results suggest that the non-random S-cone distributions can efficiently receive blurred retinal images.
\end{abstract}

Keywords: Cone Mosaic, Retina, Mathematical Model, Simulation, Eye Optics.

\section{Introduction}

In the human eye, light passes through the eye structure and refracts to form an image on the retina. Cone photoreceptors convert the retinal image into electrical signals. Image quality depends on diffraction at the pupil and aberration in the cornea and lens. Chromatic aberration also occurs due to light dispersion. This effect is larger with short wavelength light. Cones show maximum light sensitivity to either the long (L), medium (M), or short (S) wavelengths, which in turn mediate color vision. Anatomically, cones form a distinctive spatial arrangement. The S-cones have the following remarkable properties. S-cones are rare, are absent from the central fovea, and are spaced widely compared to the other cones. Since cone photoreceptors function as the input of the visual system and form a distinctive spatial arrangement on the retina, it is important to analyze how cones process information.

A cone mosaic model was recently developed and used to analyze their information processing rate [1]. In the previous study, cones were arranged on a regular grid although cones are ununiformly arranged as a cone mosaic. Thus, information in the retina is encoded based on responses from different cone types at different locations, which include spatial interaction. A realistic cone mosaic model has been developed based on the anatomical and physiological characteristics of cones [2]. However, the model omits the optical system, which should have an effect on human vision. In this study, we integrated an optics model with the cone mosaic model in order to explain the influence of human optical structures on our vision.

\section{Model}

\subsection{Eye Optics Model}

In this study, we used a schematic eye model proposed by Navarro [3]. The model is composed of a cornea and a lens. The pupil function is expressed as:

$$
P(r)=\exp \left(-0.05 R_{p}^{2} r^{2} \ln 10\right)\left[\exp i k\left(\delta_{0} W_{20} r^{2}+W_{40} r^{4}+W_{60} r^{6}+W_{80} r^{8}+\cdots\right)\right]
$$

where $0 \leq r \leq 1$ is the normalized pupil radial coordinate, $R_{p}$ is the pupil radius [mm], $\delta_{0} W_{20}$ is a defocusing factor that accounts for chromatic aberration, and $W_{40}, W_{60}, W_{80}, \ldots$ are the resulting spherical aberration coefficients. The first factor $\exp \left(-0.05 R_{p}^{2} r^{2} \ln 10\right)$ is the effective transmittance produced by the Stiles-Crawford effect.

The defocusing coefficient is calculated using the following equation [4]: 


$$
\begin{gathered}
D(\lambda)=p-\frac{q}{\lambda-c} \\
p=1.7312, q=0.63346, c=0.21410
\end{gathered}
$$

where $\lambda$ is the wavelength [m]. The spherical aberration coefficients $W_{40}, W_{60}, W_{80}, \ldots$ are estimated by polynomial fitting to ray tracing data for longitudinal spherical aberration [3].

The point-spread function (PSF) is computed by taking the Fourier transform of the pupil function. By convoluting the input image with the polychromatic point-spread function, we can calculate the retinal image in the presence of aberrations.

\subsection{Cone Mosaic Model}

We developed a mathematical cone mosaic model, based on the anatomical and physiological characteristics of the cones. Cones are concentrated in the fovea, and the cone density decreases and the diameter of the cone increases with distance from the fovea. L- and M-cones are densely and randomly distributed, while there are fewer S-cones and they are distributed non-randomly. The cone mosaic was generated with the following algorithm to reproduce the unique spatial arrangement.

1. Define the model size, total number of cones, $\mathrm{L} / \mathrm{M}$ cone ratio.

2. Determine the coordinates of each S-cone.

3. Randomly assign the coordinates of the L- and M-cones.

4. Assign a cell type (L/M/NO-CELL) to all coordinate points.

5. Generate a corresponding color for each cell and generate a photoreceptor mosaic image.

The response of cones to light was modeled using membrane dynamics equations [5]. We introduced wavelength sensitivity [6] to reproduce the responses of different cone types. The wavelength sensitivity is expressed by the following equation:

$$
\begin{gathered}
\log S=\sum_{n=0}^{6} a_{n}\left[\log \left(\frac{1}{\lambda} \frac{\lambda_{m}}{\lambda_{r}}\right)\right]^{n} \\
a_{0} \sim a_{6}:-5.2734,-87.403,1228.4,-3346.3,-5070.3,30881,-31607 \\
\lambda_{m}(m=L, M, S): 561,531,430[\mathrm{~nm}], \lambda_{r}: 561[\mathrm{~nm}]
\end{gathered}
$$

where $\lambda$ is the optical wavelength [nm]. Fig. 1 shows the wavelength sensitivity for each cone type. The three cone

types show different wavelength sensitivities.

The input image is a natural color image and used RGB inputs. The input stimulus was calculated using the following equation:

$$
I=A\left(s\left(P_{R}, \text { type }_{\text {cone }}\right) P_{R}+s\left(P_{G}, \text { type }_{\text {cone }}\right) P_{G}+s\left(P_{B}, \text { type }_{\text {cone }}\right) P_{B}\right) / 255
$$

where $A$ is scaling factor, $P_{R}, P_{G}$, and $P_{B}$ are the RGB pixel values of the input image, respectively, and $s\left(P_{R}\right.$, type $\left._{\text {cone }}\right), s\left(P_{G}\right.$, type cone $)$, and $s\left(P_{B}\right.$, type $\left._{\text {cone }}\right)$ are the maximum wavelength sensitivity of the cones. 


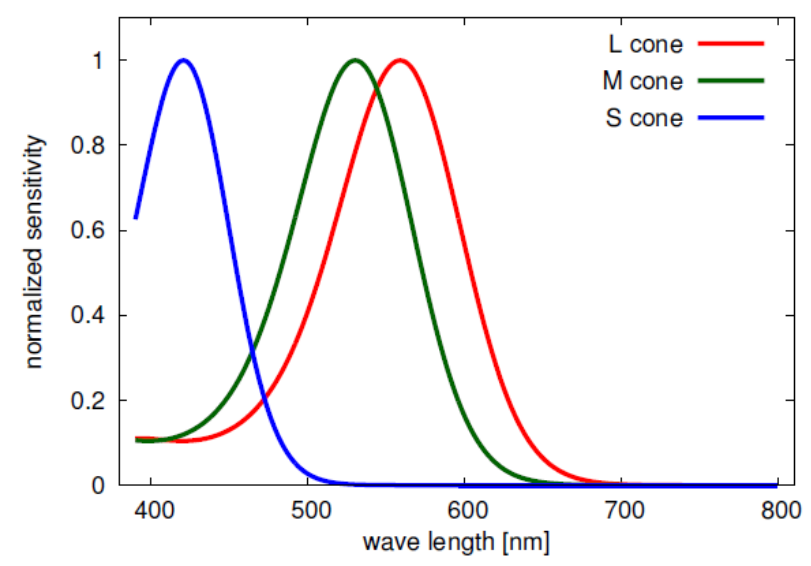

Fig. 1: Wavelength sensitivity of each type of cone.

\section{Results and Discussion}

\subsection{Characteristics of the Human Eye}

Figs. 2 and 3 show how light passes through human eye optics and strikes the retina. Short wavelength light (Fig. 2(c)) is more blurred than light with long (Fig. 2(a)) and medium (Fig. 2(b)) wavelengths. The spread for short wavelengths is $70 \%$ larger than for long wavelengths and 20\% larger than for medium wavelengths at 50\% of peak intensity.

Fig. 4 shows a generated cone mosaic (visual angle is $1^{\circ}, \mathrm{L}: \mathrm{M}=1$ ). The cone photoreceptors are concentrated in the central part of the cone mosaic (Fig. 4(a)) and have lower density in the peripheral part than the central part of the cone mosaic (Fig. 4(b)). On the other hand, the S-cones are sparsely distributed, as shown in Fig. 4(c).

\subsection{Simulation of Cone Mosaic}

Fig. 5 shows the responses of the cone mosaic. Table 1 summarizes the number of cones responding to input light. Since light spreads due to aberrations, many more cones react to input light when aberrations are present compared to a system with no aberrations. The S-cones catch broader spatial images than the L- and M-cones, because short wavelength light is blurred by chromatic aberration. It was shown that the S-cones can sample the retinal image due to their non-random distribution. The space between S-cones is much larger than that of the L- and M-cones. Chromatic aberration in the lens blurs short wavelength light. The S-cones are particularly sensitive to short wavelength light, which creates the blur seen in the retinal image. Because in normal condition the retinal image changes smoothly over space, it can be represented using few spatial samples. Thus, the theoretical exceptions and empirical observations concerning the sparse S-cone sampling mosaic agree well. The simulation results are consistent with these ideas.

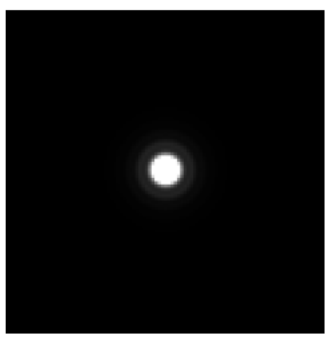

(a) $\lambda=590 \mathrm{~nm}$

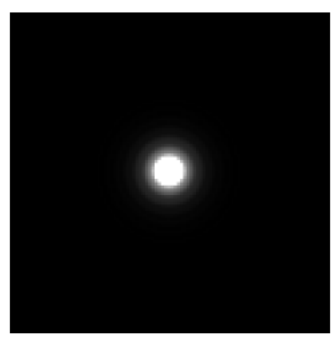

(b) $\lambda=526 \mathrm{~nm}$

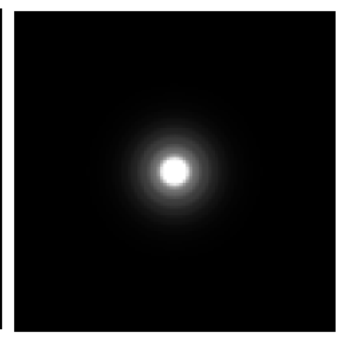

(c) $\lambda=452 \mathrm{~nm}$

Fig. 2: Light striking the retina. 


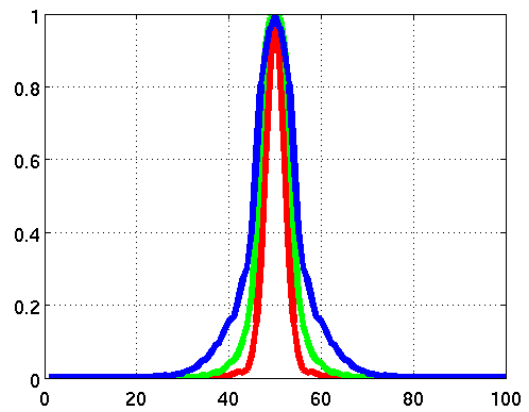

Fig. 3: One-dimensional representation of the retinal image in Fig. 2.

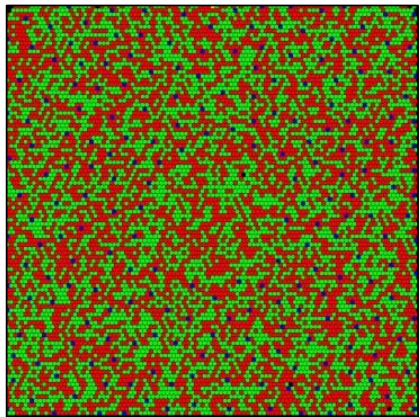

(a)

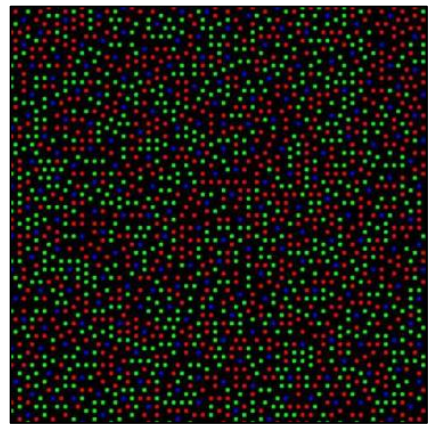

(b)

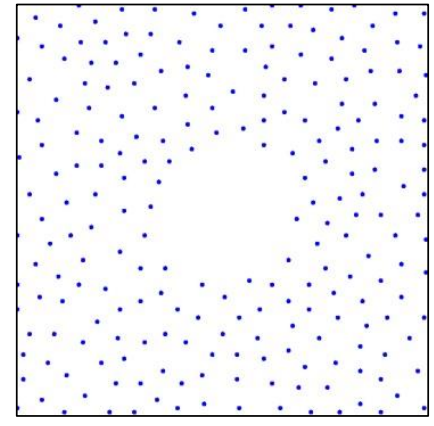

(c)

Fig. 4: A generated cone mosaic. (a) Central part of the cone mosaic, (b) peripheral part of the cone mosaic, and (c) central part of the S-cone mosaic.

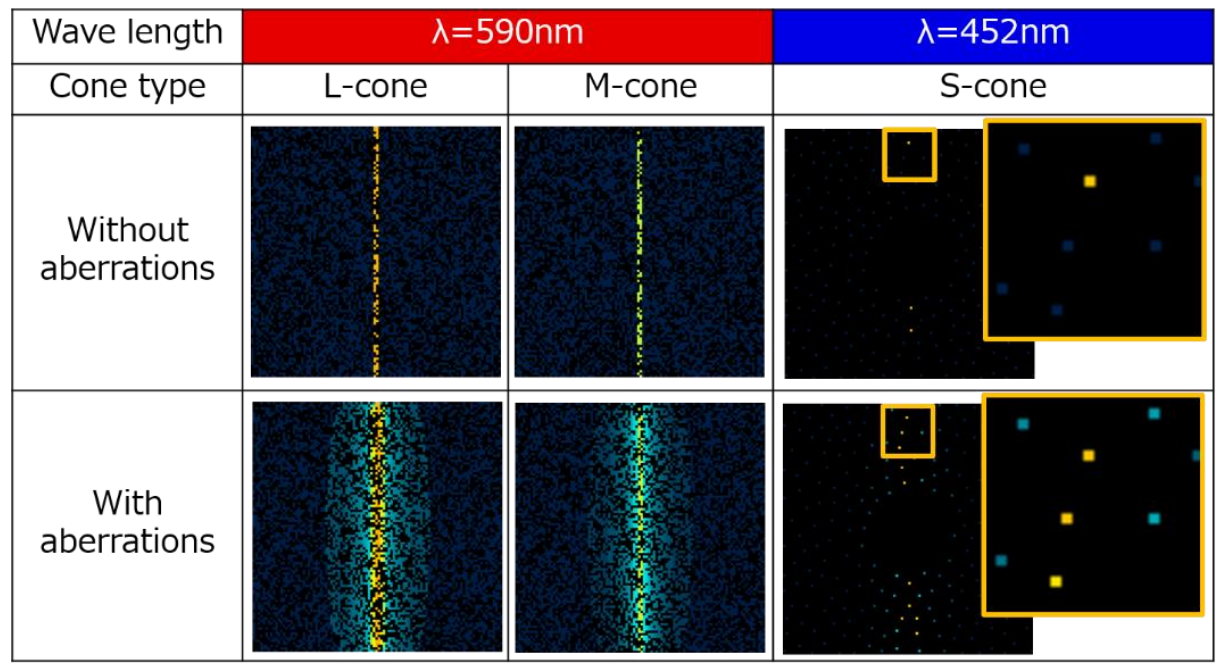

Fig. 5: Simulated mosaic response.

Table 1: The number of responded cone.

\begin{tabular}{|c|c|c|c|}
\hline Cone type & L & M & S \\
\hline Total number of responding cones & 4691 & 4768 & 224 \\
\hline Without aberrations & 96 & 100 & 3 \\
\hline With aberrations & 174 & 315 & 11 \\
\hline
\end{tabular}




\section{Conclusion}

In this study, we developed a cone mosaic model with human eye optics that can be used to analyze the photoresponse of a cone mosaic. We incorporated aberrations in the cone mosaic model by introducing a schematic eye model. As a result, it was shown that the response behavior of the cones could be reproduced with the proposed model. It was found that the Scones could catch a retinal image, despite their sparse distribution. The simulation results show that the non-random distribution of S-cones is useful for encoding retinal images and transferring information smoothly.

\section{References}

[1] P. Garrigan, C. P. Ratiff, J. M. Klein, P. Sterling, D. H. Brainard, V. Balasubramanian, "Design of a trichromatic cone array," PLoS Computational Biology, vol. 6, no. 2, pp. 1-17, 2010.

[2] N. Saito, Y. Kamiyama, "Modeling a photoreceptor mosaic based on the retinal anatomy," INCF Japan Node International Workshop: Advances in Neuroinformatics, p.19, 2015.

[3] R. Navarro, J. Santamaria, J. Bescos, "Accomodation dependent model of the human eye with aspherics," Jounal of the Optical Society of America A, vol. 2, no. 8, pp. 1273-1280, 1985.

[4] B. A. Wandell, D. H. Marimont, "Matching color images: The effects of axial chromatic aberration," Journal of the Optical Society of America A, vol. 11, no. 12, pp. 3113-3122, 1994.

[5] J. H. van Hateren, H. P. Snippe, "Simulating human cones from mid-mesopic up to high-photopic luminances," Jounal of Vision, vol. 7, no. 4, pp. 1-11, 2007.

[6] D. A. Baylor, B. J. Nunn, J. L. Schnapf, "Spectral sensitivity of cones of the monkey Macaca fascicularis," Jounal of Physiology, vol. 390, pp. 145-160, 1987. 